\title{
Foreign-Body Reaction to Orthopedic Hardware a Decade After Implantation
}

\author{
Amy Lauer, PA-C, MPAS, MS; Brian Burke Adams, MD, MPH
}

\section{PRACTICE POINTS}

- Cutaneous reactions to implantable devices occur with varying frequency and include infectious, hypersensitivity, allergic, and foreign-body reactions.

- Most reactions typically occur within the first weeks or months after implantation; however, a reaction rarely may present several years after implantation.

\section{To the Editor:}

Cutaneous reactions to implantable devices, such as dental implants, intracoronary stents, prosthetic valves, endovascular prostheses, gynecologic devices, and spinal cord stimulator devices, occur with varying frequency and include infectious, hypersensitivity, allergic, and foreign-body reactions. Manifestations have

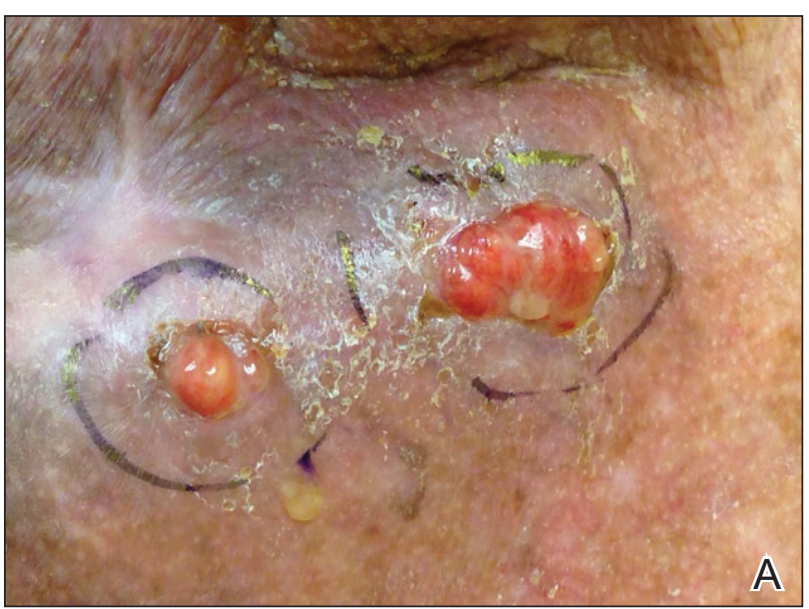

included contact dermatitis; urticarial, vasculitic, and bullous eruptions; extrusion; and granuloma formation. ${ }^{1,2}$ Immune complex reactions around implants causing pain, inflammation, and loosening of hardware also have been reported. ${ }^{3,4}$ Most reported cutaneous reactions typically occur within the first weeks or months after implantation; a reaction rarely presents several years after implantation. We report a cutaneous reaction to an orthopedic appliance almost 10 years after implantation.

A 67-year-old man presented with 2 painful nodules on the right clavicle that were present for several months. The patient denied fever, chills, weight loss, enlarged lymph nodes, or night sweats. Approximately 10 years prior to the appearance of the nodules, the patient fractured the right clavicle and underwent placement of a metal plate. His medical history included resection of

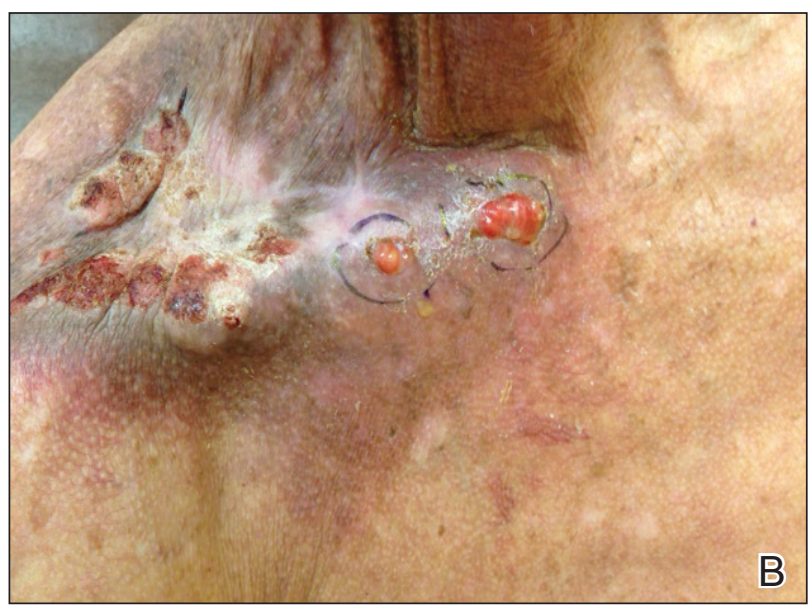

FIGURE 1. A and B, Two pink friable nodules measuring 1.5 to $2.5 \mathrm{~cm}$ in diameter and leaking serous fluid on the right clavicle at the initial presentation.

From the Department of Dermatology, Cincinnati Veterans Affairs Medical Center, Ohio. Dr. Adams also is from the Department of Dermatology, University of Cincinnati.

The authors report no conflict of interest.

Correspondence: Amy Lauer, PA-C, MPAS, MS, 3200 Vine St, Cincinnati, OH 45220 (amy.lauer@va.gov).

doi: $10.12788 /$ cutis. 0126 


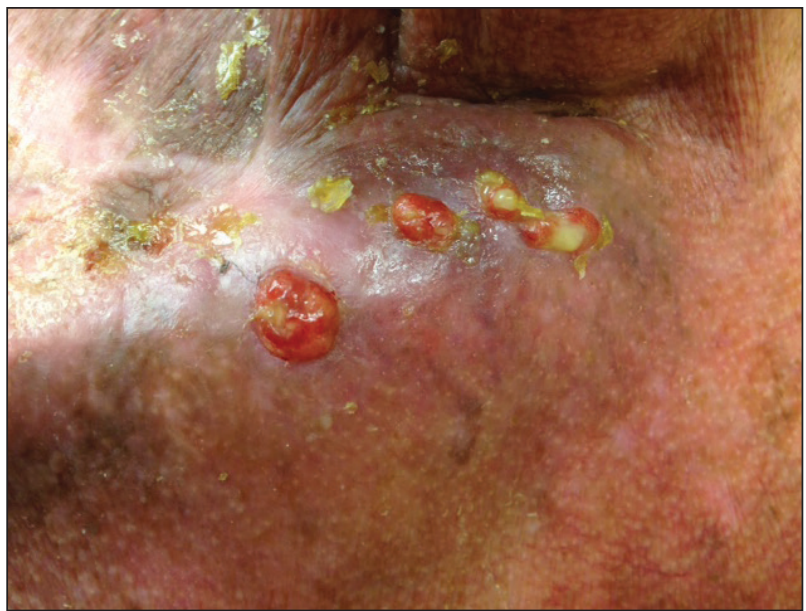

FIGURE 2. Four discrete lesions measuring 4 to $7 \mathrm{~mm}$ in diameter on the right clavicle 1 week after the initial presentation.

the right tonsil and soft-palate carcinoma with radical neck dissection and postoperative radiation, which was completed approximately 4 years prior to placement of the metal plate. The patient recently completed 4 to 6 weeks of fluorouracil for shave biopsy-proven actinic keratosis overlying the entire irradiated area.

Physical examination revealed 2 pink friable nodules measuring 1.5 to $2.5 \mathrm{~cm}$ in diameter and leaking serous. fluid within the irradiated area (Figure 1). The differential diagnosis included pyogenic granuloma, cutaneous recurrent metastasis, and atypical basal cell carcinoma. A skin biopsy specimen showed hemorrhagic ulcerated skin with acute and chronic inflammation and abscess.

The patient presented for excisional biopsy of these areas on the right medial clavicle 1 week later. Physical examination revealed the 2 nodules had decreased in diameter; now, however, the patient had 4 discrete lesions measuring 4 to $7 \mathrm{~mm}$ in diameter, which were similar in appearance to the earlier nodules (Figure 2). He reported a low-grade fever, erythema, and increased tenderness of the area.

Underlying loosened orthopedic hardware screws were revealed upon punch biopsies of the involved areas (Figure 3). Wound cultures showed abundant Staphylococcus aureus and moderate group B Streptococcus; cultures for Mycobacterium were negative. The C-reactive protein level was elevated $(5.47 \mathrm{mg} / \mathrm{dL}$ [reference range, $\leq 0.7 \mathrm{mg} / \mathrm{dL}]$ ), and the erythrocyte sedimentation rate was increased ( $68 \mathrm{~mm} / \mathrm{h}$ [reference range, $0-15 \mathrm{~mm} / \mathrm{h}$ ]). A complete blood cell count was within reference range, except for a mildly elevated eosinophil count (6.7\% [reference range, $0 \%-5 \%])$. The patient was admitted to the

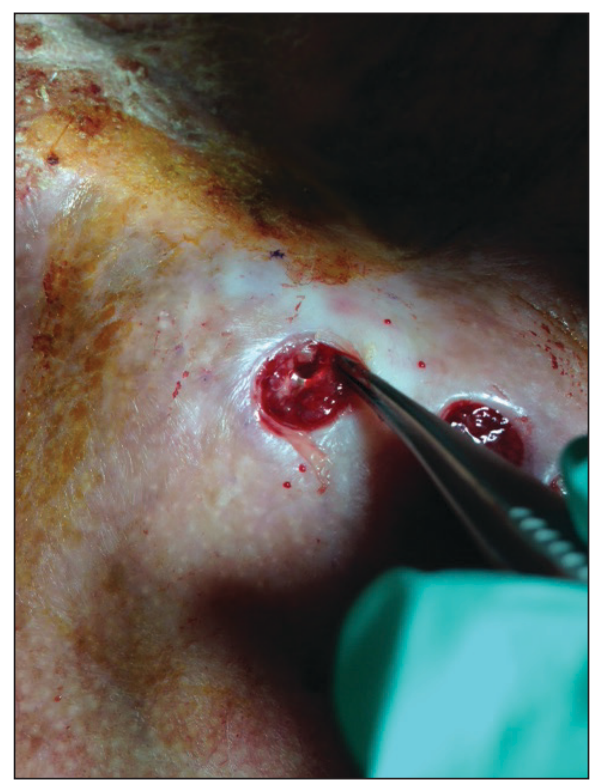

FIGURE 3. Underlying loosened orthopedic hardware screws were revealed upon punch biopsy.

hospital, and antibiotics were started. Two days later, the orthopedic surgery service removed the hardware. At 3-week follow-up, physical examination revealed near closure of the wounds.

Cutaneous reactions to orthopedic implants include dermatitis, as well as urticarial, vasculitic, and bullous eruptions. Immune complex reactions can develop around implants, causing pain, inflammation, and loosening of hardware. ${ }^{1,3}$ Most inflammatory reactions take place within several months after implantation. ${ }^{3}$ Our patient's reaction to hardware 10 years after implantation highlights the importance of taking a detailed and thorough history that includes queries about distant surgery.

\section{REFERENCES}

1. Basko-Plluska JL, Thyssen JP, Schalock PC. Cutaneous and systemic hypersensitivity reactions to metallic implants. Dermatitis. 2011;22:65-79.

2. Chaudhry ZA, Najib U, Bajwa ZH, et al. Detailed analysis of allergic cutaneous reactions to spinal cord stimulator devices. J Pain Res. 2013;6:617-623.

3. Huber M, Reinisch G, Trettenhahn G, et al. Presence of corrosion products and hypersensitivity-associated reactions in periprosthetic tissue after aseptic loosening of total hip replacements with metal bearing surfaces. Acta Biomater. 2009;5:172-180.

4. Poncet-Wallet C, Ormezzano Y, Ernst E, et al. Study of a case of cochlear implant with recurrent cutaneous extrusion. Ann Otolaryngol Chir Cervicofac. 2009;126:264-268. 